\title{
UN MARCO DE CUALIFICACIONES PARA LA CAPACITACIÓN Y LA CERTIFICACIÓN DE COMPETENCIAS LABORALES EN CHILE
}

\section{El mundo del trabajo en Chile: carencias y desafíos}

Avanzada ya la segunda década del presente siglo, Chile pareciera encaminarse decididamente a ser considerado un país con una economía desarrollada, como se desprende al observar la progresión de sus indicadores macroeconómicos. No obstante las buenas cifras económicas agregadas, se requiere bastante más que eso para ser un país desarrollado. En el camino se deben abordar distintos desafíos que se encuentran tanto en los ámbitos de la misma economía (la alta dependencia del cobre y la poco diversificada matriz productiva, por ejemplo) como en otros sistemas sociales cuya forma de funcionamiento es considerada relevante, como los sistemas educacionales, del trabajo, previsional o de salud u otros ámbitos centrales de la vida en sociedad: la densidad organizacional de la sociedad civil, el desarrollo sustentable y el ejercicio de la ciudadanía, etcétera.

Por su parte, el proceso de globalización ha permitido no solo una integración económica con muchos países, sino que también ha posibilitado la observación comparativa de nuestra sociedad y economía con el resto del mundo, especialmente a partir de la integración de Chile a la Organización para la Cooperación y el Desarrollo Económico (OCDE). En su último informe (OCDE, 2013) dicha organización plantea que a medida que la economía avanza el bienestar de las personas en Chile debiera verse reflejado en la expansión del acceso a una educación de calidad, salud pública y programas de protección social eficientes. En lo referente al mundo del trabajo sugiere que se debe avanzar en hacer más equitativo el ingreso al mercado laboral para las mujeres y jóvenes. Para 2012 las tasas de participación en la fuerza de trabajo chilena para ambos segmentos 
de personas eran inferiores al promedio de los países de la OCDE y en términos de desempleo presentaba una tendencia similar, ya que la tasa de desempleo femenina es superior al promedio general y la tasa de desempleo juvenil prácticamente la triplica. Un dato aún más preocupante es que alrededor del 15\% de los jóvenes y 30\% de las jóvenes entre 15 y 29 años no está ni en el mercado laboral ni tampoco estudiando. Finalmente, un contingente importante de personas que se encuentra trabajando posee bajos niveles de competencias para desempeñarse en el mundo laboral, por lo que se encuentra en los márgenes del sistema y no posee condiciones laborales que sean asimilables a las de «trabajo decente» establecidas por la Organización Internacional del Trabajo (Somavía, 1999).

En el informe de la OCDE mencionado también se establece que en Chile más del 20\% de las personas ocupadas está en la informalidad, esto es, sin contrato de trabajo y sin acceso a los sistemas previsionales y de salud. Esto no solo refleja los diferenciales de competencias que es posible encontrar en las personas dentro del mundo del trabajo, sino que también los diferenciales de desarrollo y modernidad entre los distintos sectores productivos y diferentes tamaños de empresa. En la práctica, conviven sectores cuyas empresas más grandes son de clase mundial, como por ejemplo en la minería, con sectores de baja productividad y desarrollo tecnológico, como la pequeña agricultura $y$, en general, las micro y pequeñas empresas. Del mismo modo la demanda también es diversa, lo que muestra un mercado del trabajo heterogéneo que pareciera no estar en condiciones de absorber el incremento de la oferta de trabajo que produciría el ingreso de más jóvenes y mujeres al mercado laboral.

Mirado desde la estructura productiva, un sector de la economía ofrece puestos de trabajo con altas productividades asociadas, mientras que otro, el mayoritario, entrega puestos de trabajo de bajas retribuciones salariales. Esto claramente es una consecuencia de la diferenciada estructura productiva del país por sector y tamaño de empresa, y su dinámica de cambio depende tanto de la inserción de las empresas en las cadenas de valor nacionales o internacionales como de las políticas de fomento productivo impulsadas desde el Estado. 
Aun cuando se fuera avanzando en un mejoramiento de la calidad de los puestos de trabajo ofrecidos, subsiste un desafío por el lado de la oferta. Las diferencias de productividad que se observan en el mercado no solo son consecuencia de factores estructurales por el lado de la demanda, sino que también reflejan las bajas competencias adquiridas que tienen sectores importantes de la población laboral para enfrentar el mundo del trabajo. De acuerdo con el estudio del Centro Microdatos (2013) un 44,3\% de la población adulta se encuentra en situación de analfabetismo funcional en textos, $42 \%$ en documentos y $51 \%$ en el área cuantitativa. Comparado con los datos del año 1998, prácticamente no hubo avances, salvo que un $7 \%$ de la población pasó de tener Nivel 1 a Nivel 2 en cuanto a documentos, según la metodología de clasificación especificada en el mismo estudio.

Por otra parte, en el informe de la OCDE ya citado se establece que el capital humano pareciera ser uno de los obstáculos para el aumento de la productividad de las empresas en Chile. En efecto, si bien ha mejorado sustancialmente el indicador que mide la cantidad de personas en el mercado laboral que ha terminado su enseñanza media, pasando de 56\% para la generación de 55 a 64 años en 2011 a $88 \%$ para la generación desde los 24 a 34 años para el mismo año, persiste en el mercado del trabajo un contingente importante de personas que no ha conseguido su licencia de enseñanza media.

Las deficiencias observadas en la oferta y la demanda de trabajo se ven reforzadas por la opacidad y las asimetrías de información existentes, tanto en el mercado del trabajo como en el de las alternativas que permiten mejorar los conocimientos, habilidades y destrezas de las personas en el mundo laboral. No hay mecanismos ni instrumentos suficientemente adecuados que permitan a las personas visibilizar sus competencias en el mundo laboral, ni tampoco instrumentos que permitan a cualquier empleador establecer las brechas entre estas competencias y la productividad y desempeño requerido, ni para diseñar e implementar programas de formación orientados a desarrollarlas. 
Esta disociación se refuerza al observar la poca correspondencia entre los requerimientos del mundo del trabajo y la formación terciaria. Un informe realizado por Kis y Field (2009) indica que los vínculos entre los empleadores y el sector de la educación terciaria son relativamente débiles. Pareciera no existir un sistema bien desarrollado que permita a los empleadores comunicar sus necesidades a las instituciones de educación superior, lo que puede deberse a razones culturales de dos mundos que operan en paralelo, con pocas interconexiones sistémicas, debido a la ausencia de una institucionalidad que los vincule y también estructurales, las que se grafican en la composición relativamente inflexible de la educación superior. Esto sería válido también para el ámbito de la formación técnico profesional.

Desde el Estado existen instrumentos que debiesen apuntar a solucionar estos desafíos, pero las evaluaciones realizadas muestran que el uso de estos no ha estado focalizado en las personas de más bajos ingresos, como el sistema de capacitación laboral (PNUD, 2011), o son aún incipientes, como el sistema de certificación de competencias. Sin embargo, la experiencia internacional muestra que «un sistema ampliamente reconocido de certificación de competencias puede mejorar la empleabilidad y productividad a través de incentivar la capacitación laboral y la certificación de competencias» (OCDE, 2013: 67).

Ambos sistemas, el de capacitación y formación laboral y el de certificación de competencias forman parte de lo que se denominan «políticas activas de empleo», las que están destinadas a disminuir los costos personales, familiares y sociales que se producen a partir del desempleo, el subempleo y los empleos de baja productividad. Con la implementación de este tipo de políticas, mediante instrumentos y programas públicos, se busca mitigar los efectos de las brechas formativas que inciden en el ingreso, permanencia y trayectoria laboral de las personas, esto es, asimetrías de información que no solo están determinadas por el conocimiento relativo que cada persona tiene del mercado del trabajo y sus proyecciones. También se tiene como meta ampliar las redes sociales en las que participan las personas, las que les generan mayores o menores perspectivas de desarrollo, y disponer de 
instrumentos que provean financiamiento para trayectorias formativas que permitan la obtención de empleos de mayor productividad y mejor remunerados.

En la actualidad las políticas activas de empleo en el mundo desarrollado han ido convergiendo cada vez más hacia la operación de sistemas de formación laboral permanentes, integrados con el mundo formativo técnico y técnico profesional, que permitan diseñar itinerarios formativos y trayectorias laborales a lo largo de la vida por distintas combinaciones de formación y desempeño laboral. Esto opera basado en metodologías flexibles que hacen posible la formación laboral de forma paralela con la ocupación de un puesto de trabajo, la existencia de equivalencias o «pasarelas» entre el mundo formativo y la experiencia laboral, el reconocimiento de los aprendizajes previos y la certificación de competencias, de manera que las personas dispongan de la institucionalidad, los instrumentos, los mecanismos de financiamiento y la información necesaria para el diseño de sus trayectorias formativas y laborales.

Lo anterior necesariamente requiere dotar a la sociedad de una institucionalidad que permita, por una parte, el funcionamiento de sistemas capacitación laboral y de certificación de competencias relacionados y articulados y, por otra, la integración de estos sistemas con el mundo formativo, en particular con la formación técnica y técnico profesional.

\section{Un marco de cualificaciones como instrumento para articular la formación laboral y la certificación de competencias}

En el contexto recién descrito adquiere sentido un marco de cualificaciones específico para los ámbitos de la formación laboral y la certificación de competencias laborales, al ser un instrumento que permite desarrollar, organizar y reconocer los conocimientos, habilidades y destrezas que son demandadas para desempeñarse en el mundo laboral, ordenándolas en niveles continuos y trazándolas sobre la base de descriptores previamente determinados. 
Un marco de este tipo considera tanto las credenciales del sector educativo como las del mundo laboral y se define como un «instrumento para el desarrollo, clasificación y reconocimiento de habilidades, conocimiento y competencias a lo largo de un continuo de niveles acordados, estructurado a partir de resultados de aprendizaje» (Tuck, 2007:2). Con ello se busca la flexibilidad de las trayectorias, facilitando la articulación entre el espacio educacional y el laboral. Si se considera que un marco «es la ruta por la que un país trae la educación y la capacitación juntas en un sistema unificado» (Tuck, 2007: 3), en un futuro escenario en el que se cuente con un marco nacional de cualificaciones, es posible considerar que el presente proyecto generará como producto un submarco que integre las cualificaciones obtenidas mediante la formación laboral, la certificación de competencias y la formación técnica que se vincula con ellas para distintos niveles de cualificaciones.

Lo anterior implica establecer los niveles de cualificaciones e incorpora no solo aquellas logradas principalmente por la formación laboral o la certificación de competencias, sino también aquellas provenientes de la formación técnica, técnico profesional o profesional, en sus distintos niveles. Cada nivel del marco tiene asociadas cualificaciones que pueden ser acreditadas mediante algún tipo de credencial, lo que posibilita reconocer los aprendizajes de cualquier tipo y visibilizarlos socialmente y en el mundo laboral, lo que hoy no es posible para un importante contingente de la fuerza de trabajo en Chile.

Lo anterior adquiere especial relevancia si se considera que el Estado financia, con distintos instrumentos, la operación de un vasto sistema de capacitación laboral y de un incipiente sistema de certificación de competencias laborales. La revisión de la experiencia internacional muestra, como se detalla más adelante, que es el Estado quien aborda el diseño y construcción de marcos de cualificaciones, ya que posee ciertas características de bien público que los distintos actores del mundo del trabajo o la formación no están dispuestos a financiar. Una de ellas es el necesario nivel de generalidad de las cualificaciones y niveles, de manera que reflejen conocimientos, habilidades y destrezas que puedan aplicar a distintos ámbitos y 
sectores laborales para que las personas puedan diseñar trayectorias formativas y laborales, teniendo como referencia el contexto global del mundo del trabajo. Disponer de un marco de cualificaciones permite ordenar las acciones públicas en los ámbitos de la formación laboral y la certificación de competencias, orientando el gasto público hacia aquellas acciones que permitan que las personas beneficiadas obtengan las credenciales asociadas a determinados niveles de cualificaciones, poniendo el foco en los resultados por conseguir.

También es relevante la confluencia de intereses que se produce para que un marco de cualificaciones sea un instrumento útil no solo para el Estado, sino que también sea válido para los distintos actores sociales a los cuales influye en su proceso de toma de decisiones.

La utilidad de un marco de cualificaciones para las personas es que en un modelo de formación continua a lo largo de la vida permite que estas visualicen sus posibles trayectorias laborales futuras y las trayectorias de aprendizaje asociadas, al identificar claramente las cualificaciones que requiere, tanto si desea moverse dentro de un sector productivo, o bien, si quiere desplazarse hacia otro sector. También permite que las personas puedan visibilizar sus aprendizajes en el mundo del trabajo, mejorando sus posibilidades de inserción y movilidad laboral.

Para las empresas, el principal uso de un marco de cualificaciones es que les permite identificar claramente, por medio de las credenciales asociadas, el nivel de aprendizaje que tiene una persona. En el caso de quienes ya están ocupados en una empresa, facilita el diseño de programas de formación y capacitación; en el caso de personas que la empresa desee contratar, tener un marco de este tipo permite disminuir los costos de transacción y entrenamiento, al asociar directamente un cargo con las cualificaciones que se requieren.

Para las entidades de formación laboral, en tanto, disponer de un marco de cualificaciones que relacione los distintos niveles con las credenciales necesarias para demostrar las cualificaciones 
obtenidas permite el diseño articulado de programas de formación orientados tanto hacia la certificación de competencias laborales como a los diseños modulares de formación laboral que se integren con la formación técnica y técnico profesional. Ello permite a la larga transparentar la oferta formativa y relacionarla directamente con los resultados que se podrían obtener.

Aun cuando el objetivo del proyecto en el que se inserta este trabajo es el diseño de un marco acotado a la formación laboral y a la certificación de competencias, se hizo necesario realizar una revisión de la experiencia internacional y nacional en cuanto a la elaboración de marcos nacionales de cualificaciones, de manera de obtener elementos para un diseño específico que luego permitiese su integración a un futuro marco nacional propio. Los resultados de esa indagación se presentan a continuación.

\section{Situación actual de los marcos de cualificaciones}

Los marcos de cualificaciones surgen en la década de los noventa en los países anglosajones con el objetivo de facilitar la articulación y flexibilización entre diferentes subsistemas de formación, integrándolos en el reconocimiento de cualificaciones para el aprendizaje a lo largo de la vida.

Este concepto nace debido a las nuevas formas de hacer y relacionarse que se generalizan a partir de la globalización y los avances tecnológicos y del conocimiento, lo que conlleva considerables exigencias acerca de la productividad de la fuerza de trabajo en las empresas y, por lo tanto, demanda que los individuos estén en constante aprendizaje y actualización de sus competencias.

Posteriormente, los marcos de cualificaciones penetraron con fuerza en la escena internacional, por considerarse un modelo alternativo que explica la forma en la que operan las credenciales educativas y que se basa en los resultados de aprendizaje (learning outcomes), más que en el proceso para lograrlos. El conjunto de conocimientos, habilidades y/o competencias que una persona ha adquirido y/o puede demostrar después de haber completado un 
proceso de aprendizaje es el que permite validar las credenciales obtenidas en un proceso educacional sistemático.

Actualmente, los primeros países que implementaron marcos de cualificaciones están en la fase de revisión de su construcción y ejecución. Este es el caso del Centro Europeo para el Desarrollo de la Formación Técnica que elaboró un reporte (CEDEFOP, 2010) en el que indica que varios países europeos han pasado de la fase de conceptualización temprana a la de consulta y pruebas y de allí a la etapa de adopción final. Más aún, otro informe de CEDEFOP (2011) muestra una rápida progresión en el diseño e implementación de los marcos de cualificaciones, tomando como referencia el Marco Europeo de Cualificaciones (MEC). Un ejemplo de ello es que los descriptores de nivel, en versiones iniciales o adoptados formalmente, están disponibles en 32 países de Europa. Esta rápida difusión de marcos en dicha región tiene su explicación más tangible en la necesidad de realizar homologaciones y equivalencias formativas entre los distintos países de la Unión Europea, producto de la libertad de trabajo y libre circulación de personas entre ellos.

Un primer grupo de países usan los descriptores del MEC, o bien, se alinean con estos, como por ejemplo Estonia, Chipre, Austria y Portugal. Aun así, muchos de estos países preparan una tabla aclaratoria o guías más detalladas de los descriptores de nivel, para hacer operativos sus marcos. Croacia, Grecia, Malta y Eslovaquia están cercanamente alineados con el MEC, parten de los pilares de conocimiento, habilidades y competencias ${ }^{5}$, pero introducen algunos cambios en el detalle de los descriptores.

Un segundo grupo de países ha cambiado y parafraseado la tercera columna de «competencia» del MEC. Así, mientras estos incluyen la autonomía y la responsabilidad como elementos clave en sus descriptores, tienden a incorporar dimensiones adicionales como competencias de aprendizaje, competencias comunicativas/ sociales/profesionales. En Finlandia se han agregado aspectos como

5 El concepto de «competencia» asume distintas acepciones según la tradición y cultura del país que lo adopta. 
el emprendimiento y el lenguaje. Esto puede verse como una manera de incluir y hacer explícita la dimensión de competencias clave y habilidades para el aprendizaje permanente. Las competencias clave son también enfatizadas en Islandia, Malta y Noruega. La inclusión del concepto de evaluación en el marco de Polonia indica que las personas deben ser capaces de reflejar su propio conocimiento, habilidades y competencias junto con juzgar o apreciar cómo mejorarlas.

En Letonia se han incluido el análisis, la síntesis y la evaluación, en tanto que Polonia también usa los términos de competencia social para identificar su tercer descriptor, definido como identidad/ autonomía, cooperación, responsabilidad, evaluación/conciencia de sí y de un equipo y comprensión de las implicancias de las decisiones y acciones.

El tercer y último grupo utiliza el concepto de competencia como el central, dando cuenta de las tradiciones nacionales. Acá se encuentran Bélgica, Alemania, Francia, Lituania, Hungría, Holanda y Eslovenia. La mirada de competencia en estos países es integral y holística: se considera como una habilidad para usar el conocimiento, las habilidades, actitudes y otras capacidades personales, sociales y/o metodológicas en situaciones laborales y de estudio, así como para situaciones de desarrollo profesional y personal.

A continuación se muestra una tabla resumen de los descriptores usados en los diferentes países europeos ${ }^{6}$ y Oceanía:

6 Una descripción detallada de los enfoques se encuentra en CEDEFOP (2010), CEDEFOP (2011) y CEDEFOP (2012). 
Cuadro 1: Descriptores de marcos de cualificaciones, Europa y Oceanía

\begin{tabular}{|c|c|c|c|c|c|c|c|c|c|c|c|}
\hline PAÍS & \multicolumn{11}{|c|}{ DESCRIPTORES } \\
\hline & Número & & & & & & NOMBRES & & & & \\
\hline \multirow{2}{*}{ MEC } & \multirow{2}{*}{3} & \multicolumn{3}{|c|}{ Conocimiento } & \multicolumn{3}{|c|}{\begin{tabular}{l|l} 
Habilidades &
\end{tabular}} & \multicolumn{4}{|c|}{ Competencia } \\
\hline & & Teórico & \multicolumn{2}{|c|}{ Práctico } & Cognitivas & \multicolumn{2}{|c|}{\begin{tabular}{l|l} 
S & Prácticas \\
\end{tabular}} & \multicolumn{2}{|c|}{ Autonomía } & \multicolumn{2}{|c|}{ Responsabilidad } \\
\hline Bélgica & 2 & \multicolumn{3}{|c|}{ Conocimiento/habilidad } & \multicolumn{7}{|c|}{ Contexto, autonomía y responsabilidad } \\
\hline \multirow{2}{*}{ Bulgaria } & \multirow{2}{*}{3} & \multirow{2}{*}{\multicolumn{3}{|c|}{ Conocimiento }} & \multirow{2}{*}{\multicolumn{3}{|c|}{ Habilidades }} & \multicolumn{4}{|c|}{ Competencia profesional } \\
\hline & & & & & & & & & utonomía & & ponsabilidad \\
\hline Croacia & 3 & \multicolumn{3}{|c|}{ Conocimiento } & \multicolumn{3}{|c|}{ Habilidades } & \multicolumn{4}{|c|}{ Autonomía y responsabilidad } \\
\hline Chipre & 3 & \multicolumn{3}{|c|}{ Conocimiento } & \multicolumn{3}{|c|}{ Habilidades } & \multicolumn{4}{|c|}{ Competencia } \\
\hline $\begin{array}{l}\text { República } \\
\text { Checa }\end{array}$ & 1 & \multicolumn{10}{|c|}{$\begin{array}{l}\text { Integra en la definición de competencia el conocimiento y las habilidades y la capacidad para } \\
\text { combinarlos (muy cercano a la complejidad de las actividades laborales) }\end{array}$} \\
\hline & & Conoc & niento & & & bilidade & & & & etenci & \\
\hline Dinamarca & 3 & $\begin{array}{l}\text { Tipo, cor } \\
\text { comp }\end{array}$ & $\begin{array}{l}\text { plejid } \\
\text { nsión }\end{array}$ & & $\begin{array}{l}\text { Tipo, con } \\
\text { problema, }\end{array}$ & mplejid & $\begin{array}{l}\text { lad del } \\
\text { nicación }\end{array}$ & & $\begin{array}{l}\text { Espacio de } \\
\text { responsa }\end{array}$ & $\begin{array}{l}\text { n, coo } \\
\text { d, apr }\end{array}$ & $\begin{array}{l}\text { peración y } \\
\text { rendizaje }\end{array}$ \\
\hline Estonia & 3 & Conoc & niento & & & bilidade & & & & etenci & \\
\hline Finlandia & 5 & Conocimi & & & 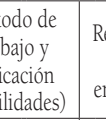 & $\begin{array}{r}\text { Respons } \\
\text { gest } \\
\text { emprenc }\end{array}$ & $\begin{array}{l}\text { sabilidad, } \\
\text { ión y } \\
\text { dimiento }\end{array}$ & & Evaluació & & $\begin{array}{l}\text { abilidades clave } \\
\text { ra el aprendizaje } \\
\text { permanente }\end{array}$ \\
\hline & & Compe & encia & profesi & onal & & & omp & apetencia pe & & \\
\hline Alemania & doble & Conocim & & & jilidades & & $\begin{array}{l}\text { Social (res } \\
\text { mpromiso, }\end{array}$ & & unilidad, & & Autonomía \\
\hline Grecia & 3 & & nocim & iiento & & & Habilidad & & & Comp & etencia \\
\hline Hungría & 4 & Conocim & & & ilidades & Actitud & & & Autonomía & jonsab & bilidad \\
\hline Islandia & 3 & Conocim & & & $\begin{array}{ll}\text { ilidades } & \mathrm{Co} \\
\end{array}$ & ompeten & ncia (son d & nicic & iones más a & is que & MEC) \\
\hline Irlanda & 3 & Conocim & & & Saber ha & acer y $\mathrm{h}$ & abilidades & & & ompet & tencia \\
\hline Latvia & 3 & Conc & imien & & & Habi & ilidades & & & ompet & tencia \\
\hline & & Cara & terísti & cas de 1 & a actividad & & & & Tipo de & encia & \\
\hline Lituania & 3 & $\begin{array}{l}\text { Complejida } \\
\text { las activid }\end{array}$ & & $\begin{array}{l}\text { utonon } \\
\text { de las } \\
\text { tividac }\end{array}$ & \begin{tabular}{l|l} 
nía & Variabilid \\
les & las activic
\end{tabular} & $\begin{array}{l}\text { idad de } \\
\text { ridades }\end{array}$ & Func & & & & General \\
\hline & & Con & imien & & & & bilidades & & & Compe & tencia \\
\hline Malta & 3 & $\begin{array}{l}\text { Más result } \\
\text { comprensi }\end{array}$ & & $\begin{array}{l}\text { apren } \\
\text { ocimier }\end{array}$ & $\begin{array}{l}\text { dizaje que se } \\
\text { ato aplicado y } \\
\text { nabilidades de }\end{array}$ & $\begin{array}{l}\text { e especif } \\
\text { y comp } \\
\text { de apren }\end{array}$ & $\begin{array}{l}\text { fican para } \\
\text { orensión, h } \\
\text { dizaje, aut }\end{array}$ & & $\begin{array}{l}\text { ivel en rel } \\
\text { ades de cor } \\
\text { ía y respon }\end{array}$ & $\begin{array}{l}\text { con: ce } \\
\text { ación, } \\
\text { dad }\end{array}$ & $\begin{array}{l}\text { onocimiento y } \\
\text { habilidades de }\end{array}$ \\
\hline & & Conocim & & & & & & bilid & dades & & \\
\hline Holanda & $3+5$ & $\begin{array}{r}\text { Conocim } \\
\text { aplica }\end{array}$ & & & $\begin{array}{l}\text { Resolución de } \\
\text { problemas }\end{array}$ & & $\begin{array}{r}\text { Aprendiza } \\
\text { desarrol }\end{array}$ & & De info & & $\begin{array}{c}\text { De } \\
\text { comunicación }\end{array}$ \\
\hline Noruega & 3 & Conocim & & & & abilidade & & & & tencia & general \\
\hline Polonia & 3 & Conocim & & & & abilidade & & & & tencia & a social \\
\hline Portugal & 3 & Conocim & & & & abilidade & & & & ctitud & \\
\hline Eslovenia & 3 & Conocim & & & & abilidade & & & & npeter & ncia \\
\hline España & 3 & Conocim & & & Habilidade & les y cap & pacidades & & & npeter & ncia \\
\hline Turquía & 3 & Conocim & & & & abilidade & & & & npeter & ncia \\
\hline UK & 4 & Síntes & & & $\begin{array}{l}\text { onocimiento y } \\
\text { comprensión }\end{array}$ & & Apli & 100 & y acción & & $\begin{array}{l}\text { tonomía y «dar } \\
\text { cuenta» }\end{array}$ \\
\hline Escocia & 5 & $\begin{array}{l}\text { Conocimi } \\
\text { comprer }\end{array}$ & & & Práctica & $\begin{array}{r}\text { Habilid } \\
\end{array}$ & $\begin{array}{l}\text { dades cogn } \\
\text { genéricas }\end{array}$ & & $\begin{array}{r}\text { Comunica } \\
\text { y habil } \\
\text { numé }\end{array}$ & $\begin{array}{l}\text { TICS } \\
\text { s }\end{array}$ & $\begin{array}{c}\text { Autonomía, } \\
\text { «dar cuenta» } \\
\text { y trabajo con } \\
\text { otros }\end{array}$ \\
\hline & & & & & OCEAI & ANÍA & & & & & \\
\hline $\begin{array}{l}\text { Nueva } \\
\text { Zelandia }\end{array}$ & 3 & Conocim & & & & bilidade & & & Aplica & $\begin{array}{l}\text { de con } \\
\text { bilidac }\end{array}$ & $\begin{array}{l}\text { ocimiento y } \\
\text { des }\end{array}$ \\
\hline Australia & 3 & Conocin & & & & bilidade & & & Aplica & $\begin{array}{l}\text { de con } \\
\text { bilidac }\end{array}$ & $\begin{array}{l}\text { ocimiento y } \\
\text { des }\end{array}$ \\
\hline
\end{tabular}


La tendencia observada a redefinir la tercera columna de los descriptores puede ser vista como un reflejo práctico de que las recomendaciones del MEC operan en dos diferentes definiciones de competencia. Mientras el concepto de competencia usado en los descriptores es limitado a autonomía y responsabilidad, la definición general apunta a relacionar la competencia con la aplicación del conocimiento, las habilidades y las competencias personales, metodológicas y profesionales. La redefinición de la tercera columna puede considerarse como un esfuerzo para integrar lo que muchos han caracterizado como una incoherencia en los descriptores del MEC. La solución simple a esto es, pues, introducir indicaciones más estrictas y precisas: esto se ejemplifica con responsabilidad e independencia (Holanda), competencia general (Noruega) y competencia social (Polonia). Lo que se puede mencionar con certeza es que todos los países que han ajustado la tercera columna concuerdan que responsabilidad y autonomía son factores clave, relevantes e importantes para describir los niveles de cualificaciones y asentarlas en el mundo del trabajo.

En Latinoamérica, de acuerdo con un estudio realizado por Eurosocial (2012), se destacan la experiencia de Colombia y Brasil, aun cuando no cuentan con marco de cualificaciones nacional. En Colombia se ha realizado un ordenamiento de los perfiles ocupacionales, generándose una buena base para la creación e implementación de un marco de este tipo a futuro, así como también se encuentra en operación un organismo que regula los requerimientos del mercado laboral y del mundo de la capacitación (Servicio Nacional de Aprendizaje-SENA). Mientras, en Brasil se ha implementado un organismo similar al de Colombia y el Ministerio de Educación ha potenciado y fortalecido la educación técnico profesional mediante mejores programas de estudio.

Como una forma de profundizar en aspectos particulares de esta revisión, a continuación se presentan tres experiencias asociadas al diseño e implementación de un marco nacional de cualificaciones que pueden entregar aprendizajes y datos de utilidad para nuestro país. 
El primero de estos casos es Colombia. Como ya se señaló, fue uno de los primeros países latinoamericanos en instalar e institucionalizar diversos mecanismos que han generado un contexto óptimo para la implementación de un marco nacional de cualificaciones. Si bien este país aún no cuenta con él, resulta relevante conocer la clasificación ocupacional desarrollada por SENA, pues muestra una metodología y estructura similar a lo que sería un futuro marco de cualificaciones.

Respecto de los elementos institucionales necesarios para un marco nacional de cualificaciones $y$, particularmente para un marco de la formación laboral y certificación de competencias, Colombia cuenta desde 2003 con el SENA, institución a cargo de ambos sistemas mencionados. Entre sus características cabe resaltar la existencia de mesas sectoriales (similares a los skill councils de otros países), instancias en las que se proponen políticas de formación y cualificación de las personas y que cuentan con una alta presencia del sector productivo y credibilidad en los ámbitos público y privado ${ }^{7}$.

Uno de los instrumentos de SENA es su Catálogo de ocupaciones, las que se ordenan en torno a dos criterios. Uno de ellos son los niveles de cualificación, que corresponde a una jerarquía conformada por cuatro niveles que se definen tanto por la complejidad de las funciones, el nivel de autonomía y responsabilidad en el desempeño de la ocupación en relación con otras, como por la cantidad, tipo y nivel de formación requeridos para su desempeño.

Un punto destacable de esta experiencia es que los criterios utilizados para la definición de los niveles en el Catálogo de ocupaciones coinciden plenamente con los descriptores usados en los marcos nacionales de cualificaciones alrededor del mundo, en especial con la «tercera columna» de ellos. Otro elemento relevante es la institucionalidad desarrollada en Colombia, la que no solo facilita el avance hacia la instalación de un marco de esta naturaleza, sino que además cuenta con legitimidad y credibilidad entre los diversos actores.

7 Ver más información en el Observatorio Nacional y Ocupacional Colombiano. Disponible en: observatorio.sena.edu.co/cno.html 
En ese mismo sentido, la existencia y validez de los consejos sectoriales, tanto para el ámbito educativo como laboral, son un aspecto a tomar en cuenta a la hora de considerar un diseño para nuestro país.

El segundo caso es Portugal, que cuenta con un marco nacional de cualificaciones de carácter amplio desde el 2010, incluyendo las cualificaciones de nivel superior en un marco más detallado, pero que está integrado al primero y que se considera como una herramienta para reformar la educación y la capacitación portuguesa. Su desarrollo forma parte de un programa más ambicioso que incluye la Iniciativa de nuevas oportunidades (NOI) ${ }^{8}$ y la Agenda para la reforma de la formación técnica, que tiene como objetivo elevar el bajo nivel de cualificación de la población?

Los tres objetivos principales de estas medidas consideraron:

- Reforzar los itinerarios técnicos/tecnológicos como opciones reales para los jóvenes, de modo de combatir la deserción, implementando un sistema de certificación doble: académica y técnica.

- Subir el nivel de educación y cualificación de la población adulta por medio de la combinación de educación y capacitación para adultos y la generalización de procesos de reconocimiento y certificación de aprendizajes y habilidades, ofreciendo oportunidades tanto en el campo general como profesional.

- Promover el logro de la educación secundaria (12 años) como el nivel mínimo de cualificación.

En el marco de esta reforma se definió la creación de tres instancias institucionales:

8 NOI es un programa público en el que se pusieron en marcha alrededor de 450 Centros de Nuevas Oportunidades para implementar la iniciativa. Estos centros han registrado un récord de 1,6 millones de matriculaciones, y han superado la impresionante cifra de 430.000 certificaciones (equivalente $\mathrm{al} 9^{\circ}$ y $12^{\circ}$ grado). Aun así, el número de centros recientemente se ha reducido a 200 y la relación entre la certificación que ofrecen y el aumento de salario o el empleo de los estudiantes exitosos se está investigando.

9 De acuerdo con los datos de Eurodata, en 2010 el 31,9\% de la población había cursado al menos la enseñanza secundaria superior. Se considera que alrededor de $72 \%$ de la mano de obra se encuentra por debajo de los estudios secundarios, o alrededor de 3,5 millones de adultos de acuerdo con el Censo de Población 2001. 
1. La Agencia Nacional para la Cualificación, encargada principalmente de la gestión del sistema.

2. El Catálogo Nacional de Cualificaciones, que tiene 235 cualificaciones para 39 áreas de educación y capacitación, definiendo para cada una de ellas un perfil ocupacional, un estándar de capacitación (que permite obtener una doble certificación) y un estándar para el reconocimiento, validación y certificación de competencias. El proceso de actualización liderado por la Agencia es apoyado por 16 consejos sectoriales, los que buscan la interacción y cooperación entre diversos actores para asegurar que las cualificaciones se ajustan a las demandas del mercado laboral.

3. El sistema de reconocimiento, validación y certificación de aprendizajes no formales e informales, que refiere a los estándares que son parte del mencionado catálogo y que se integra al marco nacional de cualificaciones y que tiene como meta promover la formación técnica ${ }^{10}$.

Respecto de las características del marco nacional de cualificaciones propiamente tal se adoptó una estructura de ocho niveles, la que cubre todas las cualificaciones generadas en varios sectores de actividad y en contextos de educación y capacitación (formal, no formal e informal). Se asumieron igualmente dos descriptores del marco europeo: conocimientos y habilidades, planteando como tercer descriptor las actitudes, en tanto que el concepto de competencia ${ }^{11}$ se definió de manera general dentro del Sistema Nacional de Cualificaciones. Estos tres descriptores se usaron para definir los objetivos de aprendizaje tanto de la formación general, como de la secundaria superior, en sus diversas áreas.

Destacable en esta experiencia es la relevancia que tuvo en su proceso el establecimiento de políticas coordinadas y coherentes,

10 Este sistema está conformado por dos procesos principales: en primera instancia el reconocimiento educacional, para mejorar el nivel educacional de adultos que no tienen su educación básica o secundaria; y, en segundo término, el reconocimiento técnico para adultos que no tienen cualificaciones técnicas en sus áreas ocupacionales. Los adultos pueden acceder a ambos certificados y estos tienen el mismo valor que los obtenidos en la educación formal y en la capacitación.

11 Capacidad reconocida para movilizar conocimiento, habilidades y actitudes en un contexto de trabajo, de desarrollo profesional o desarrollo educacional y personal. 
instalando diversas formas institucionales que aprovecharon la normativa y legislación existente, las que se reconocen como una base sólida para ir afinando la relación entre las cualificaciones existentes y los niveles del marco. Aun cuando no se ha evaluado el impacto de las medidas en términos de empleabilidad y mejora de las cualificaciones de la población, se ha reconocido en el contexto europeo el desarrollo de procesos de consulta pública, los que tuvieron como foco promover el reconocimiento de aprendizajes previos y que mostraron la capacidad para estimular un activa adquisición de formación técnica y capacitación por parte de las personas, mejorando su movilidad laboral y educativa.

Finalmente, el desarrollo de una estrategia de doble certificación (tanto educativa como laboral), en algunos de los niveles del marco nacional de cualificación, resulta como un punto interesante a considerar al momento de pensar en la implementación de un marco nacional en Chile.

Por último, resulta relevante destacar el caso de Nueva Zelandia. Como datos de contexto se debe considerar que la población de este país es un poco mayor a los cuatro millones de personas, la tercera menor en la Organización para la Cooperación Económica y el Desarrollo (OCDE) y tiene la cuarta economía más pequeña entre los 30 países que componen dicha organización. Debido a su tamaño y por tratarse de un país aislado con una baja densidad poblacional, la mayor parte de las personas trabaja en industrias relacionadas con la agricultura y aunque esta ha mantenido su importancia durante los años, nuevos sectores relacionados con el área de servicios han aumentado su relevancia (por ejemplo, finanzas, turismo y salud, entre otros).

Como una manera de responder a los cambios en la actividad económica local y global, a partir de la década de los ochenta se comenzaron a realizar distintas reformas que desembocaron en el actual marco de cualificaciones.

La estructura de dicho marco nacional de cualificaciones cuenta con diez niveles que se diferencian por su grado de complejidad, en 
donde el nivel 1 es el menos complejo y el 10 el de mayor complejidad. Todas las cualificaciones que existen en el marco están asignadas a uno de estos niveles. Es posible incluir para una cualificación los créditos obtenidos en un nivel menor o mayor al que está asociada dicha cualificación.

Cada nivel se describe en términos de resultados de aprendizaje, usando los siguientes dominios o dimensiones comunes de progresión (descriptores): conocimiento, habilidades y aplicación ${ }^{12}$; que describen lo que se espera que una persona graduada en un determinado nivel conozca, haga y sea.

En vistas de que cada persona puede lograr los resultados de aprendizaje mediante distintas vías, se decidió establecer «estándares de logro», los que sirven como indicadores de los logros mínimos esperados para obtener una cualificación. Cada uno de ellos incluye:

- Perfiles de egreso: identifican los resultados de aprendizaje esperados para una cualificación, así como también dan nociones de lo que una persona debe saber, comprender y ser capaz de hacer cuando alcanza una cualificación.

- Itinerario educativo: identifica otras cualificaciones que la persona puede obtener después de completar una determinada cualificación. El estándar de logro debe explicitar cuando una cualificación no conduce a otros estudios.

- Itinerarios de empleo (o contribución a la comunidad): identifica las áreas en las que una persona puede estar cualificada para trabajar o la contribución que puede hacer a la comunidad.

En términos de institucionalidad, el marco nacional de cualificaciones está a cargo de la Autoridad Neozelandesa de Cualificaciones, que entre sus funciones debe desarrollar, registrar y apoyar dicho marco, así como la definición de estándares.

Cabe destacar que el marco neozelandés contempla, de manera explícita, la integración de la cultura maorí en su diseño.

12 El concepto de «aplicación» incluye responsabilidad, conductas, actitudes, atributos y competencia. 
Así, existe el Foro Maorí de Desarrollo Económico, que debe identificar potenciales nuevas cualificaciones que podrán significar un mejoramiento económico que beneficie a los maoríes. Asimismo existe el Nga Kaitühono, grupo independiente que busca asegurar que el foco del marco nacional de cualificaciones hacia el conocimiento maorí sea compatible con los valores, consistente con sus expectativas y complementario con otros procesos de validación, incluyendo aquellos establecidos por los miembros de esta cultura.

Esta es una experiencia que resulta interesante para nuestro país, debido a las similitudes en distintos aspectos de su economía con incremento en los servicios y un foco en la exportación, aun cuando se trate de una población de menor tamaño. Un aspecto igualmente relevante es la solidez de la institucionalidad necesaria para diseñar el marco y definir diversos dispositivos que permitan su implementación efectiva, de modo que pueda ser utilizado por los destinatarios del sistema. Contar con una integración étnica explícita, donde se busca respetar e integrar los valores y creencias del pueblo maorí, incluso como parte de la institucionalidad, es muy interesante, especialmente considerando la perspectiva de integración de los pueblos originarios en Chile.

Por último, resulta relevante considerar las críticas que se han realizado a los marcos de «primera generación», entre los que se incluyen las primeras versiones del neozelandés. En general se les considera más radicales, pues tendieron a especificar resultados de aprendizaje independientemente del currículo y de la pedagogía, intentando definir cualificaciones separadamente de la manera en que eran formadas (proveedores). Estos países incluyen hoy en sus marcos una visión integrada, que considera no solo lo que requiere el mundo del trabajo, sino también el cómo las cualificaciones deben ser definidas, generando indicaciones para los cambios que se requieran en el currículo y en el enfoque de aprendizaje.

\section{La experiencia chilena}

Igual que en el resto del mundo, Chile también ha puesto dentro de sus temas de discusión el desarrollo de un marco de cualificación. 
Esta conversación tiene en el país al menos una década, pero esta se ha mantenido en los ámbitos del diseño y los prototipos para sectores determinados. Destaca la experiencia del proyecto en el contexto del Programa de Mejoramiento de la Calidad y Equidad de la Educación (MECESUP) desarrollado por universidades del Consejo de Rectores de las Universidades Chilenas (CRUCH), el Programa ChileCalifica y los marcos del Instituto Nacional de Capacitación Profesional, INACAP, y el desarrollado para la industria de la minería por parte de la Fundación Chile.

Las universidades del CRUCH, impulsadas por el Programa MECESUP, presentaron en 2007 un proyecto al segundo concurso del Fondo de Innovación Académica (FIAC) con el objetivo de diseñar un marco de cualificaciones que articulara títulos y grados dentro del país, así como para vincularse con la formación educacional de otros países de Europa y EE.UU. Debido a que el sistema de educación superior chileno es desarticulado y ha tenido un proceso de masificación que ha generado la necesidad de organizar mejor la oferta, se buscaba con esta iniciativa organizar títulos y grados ofrecidos dentro del territorio nacional (MECESUP Bicentenario, 2009), así como también asegurar la calidad de la educación, sobre todo considerando que se estaba avanzado en el proceso de acreditación y en un sistema de créditos transferible entre universidades. Los resultados del proyecto permitieron proponer que el desarrollo de un marco de cualificaciones (Lemaitre, 2010) debe:

- Abordar los déficits del sistema de educación superior chileno, como la normativa que rige los títulos y grados, la transparencia de lo que significa cada uno de estos certificados en términos de logros de aprendizaje, la articulación entre los subsistemas, la relevancia y pertinencia de las titulaciones, el fortalecimiento de la calidad y la articulación de los títulos y grados con el mundo del trabajo.

- Permitir la articulación entre los sectores de la educación y capacitación, particularmente el sector de la formación técnica y la educación superior.

- Describir los niveles en términos de resultados de aprendizaje que den cuenta de lo que la persona debe saber, comprender y 
ser capaz de hacer al término del programa de estudios, o bien, de la capacitación.

- Estar acompañado de políticas públicas orientadas a fortalecer la educación técnica vocacional, bajo una perspectiva del aprendizaje a lo largo de la vida.

- Nacer de la discusión, análisis y consenso de los actores clave en el tema de las cualificaciones.

- Proponer la agregación de actitudes o competencias blandas que están siendo consideradas en los nuevos diseños curriculares en el sistema educativo superior y que son valoradas por el mundo del trabajo.

En relación con el programa ChileCalifica, se puede mencionar que el objetivo principal era establecer las bases para la instalación de un sistema de formación permanente, reduciendo las brechas entre la educación y el trabajo (Programa ChileCalifica - Universidad Técnica Federico Santa María, 2010). Para ello implementó estrategias que permitieran:

1. Vincular el sector formativo con el productivo mediante la conformación de redes.

2. Proporcionar las oportunidades de progresar a lo largo de la vida, por medio del diseño de itinerarios de formación técnica y programas curriculares conectados.

3. Promover el establecimiento de la Ley del Sistema Nacional de Certificación de Competencias Laborales (SNCCL) que permite reconocer las competencias laborales de las personas, independientemente de la forma en la que hayan sido adquiridas.

Para ello, de acuerdo con la experiencia obtenida, se propuso realizar una propuesta estratégica, conceptual y metodológica validada, que pudiera ser usada como referente para el desarrollo de un marco de cualificaciones que permitiese enfrentar los desafíos de la formación permanente en Chile. La implementación fue realizada dentro del sector minero, estableciendo los niveles y sus respectivos descriptores para la educación técnica, desde oficio hasta técnica superior. Se propuso igualmente una estructura de marco con ocho niveles con descriptores que diesen cuenta de distintas cualificaciones 
sobre la base de conocimiento, competencias comunicacionales, competencias técnicas y profesionales, autonomía y responsabilidad y habilidades. Este proyecto concluyó que:

- La construcción de un marco de cualificaciones resulta pertinente en la medida en que cumpla una función ordenadora y normativa, que se dirija al aseguramiento de la calidad, estableciendo reglas y procedimientos comunes para todas las cualificaciones y que establezca un firme control sobre la forma en la que se diseñan las mismas.

- Un marco de cualificaciones debe articular las mismas entre los diferentes segmentos educativos y formativos y las necesidades del desarrollo productivo, a fin de facilitar el avance de las personas en sus trayectos formativos.

- El desafío de construcción de un marco de este tipo requiere de una decisión política de Estado, la que va más allá de un periodo de gobierno, por sus implicancias en los intereses de los usuarios y proveedores del sistema educativo de formación y por el hecho de que el tiempo para su construcción e instalación es superior a cuatro años, periodo actualmente vigente para cualquier gobierno chileno.

La evaluación posterior de las personas vinculadas a esta experiencia y entrevistadas en la etapa previa de este trabajo es que este fue un diseño muy académico, desvinculado del sector productivo, sin participación efectiva y permanente de las empresas ni de sus trabajadores y sin vinculación real con el sector de la capacitación laboral. Todo ello implicó que el marco diseñado nunca tuvo una aplicación práctica y no fue apropiado ni usado por la industria.

El proyecto de marco de cualificaciones desarrollado por el Instituto Nacional de Capacitación Profesional, INACAP, consistió en la generación de una propuesta e implementación para el sector de administración y negocios, para ello se diseñó una propuesta de marco a nivel nacional, relacionándola luego con uno específico de INACAP que se vinculara con aquel. Para el logro de este objetivo, se constituyó un comité técnico que validara internamente las decisiones, así como también se llevó a cabo un análisis de la oferta interna de la institución y su vinculación con la enseñanza media técnico 
profesional y su sistema académico, para contar con instrumentos complementarios a un marco de esta naturaleza. Los resultados del proyecto permitieron concluir que:

- Se debe considerar tanto el sector educativo como la formación continua.

- La descripción de los niveles de aprendizaje debe ser lo bastante amplia para incluir futuras credenciales, fundamentalmente títulos.

- La oferta del sector de formación y capacitación debe ser analizada en mayor profundidad, ya que las horas de los programas no son equivalentes al nivel de aprendizaje que se logra. Puede haber programas con la misma cantidad de horas, pero que dan cuenta de diferentes credenciales.

- Es primordial contar con perfiles ocupacionales validados por los sectores económicos, de modo que permitan ir ubicando las competencias en los niveles de aprendizaje.

- Se debe contar con una mesa técnica y una validación política para la adecuada implementación de un marco, así como un sistema académico con procesos y procedimientos claros y definidos, ya que ellos fortalecen y dan sentido al marco de cualificaciones.

El último proyecto por destacar a nivel nacional es el marco de cualificaciones para el sector de la minería, realizado por la Fundación Chile junto con el Consejo Minero (Consejo de Competencias Minero, 2013). Esta iniciativa buscaba generar una articulación entre la formación y las necesidades del sector productivo, debido a los nuevos desafíos de dicho mercado laboral para su crecimiento y expansión. Se usó como base el marco de cualificaciones de Australia, incluyéndose cinco niveles, pues se tomó en cuenta solamente la formación técnica.

Cuadro 2: Descriptores de marcos de cualificaciones, Chile

\begin{tabular}{|c|c|c|c|c|}
\hline & Número & \multicolumn{3}{|c|}{ NOMBRES } \\
\hline $\begin{array}{c}\text { Minería } \\
\text { Fundación Chile }\end{array}$ & 3 & Conocimiento & Habilidades & Contexto de aplicación \\
\hline
\end{tabular}

Los perfiles fueron ubicados de acuerdo con los procesos principales de la industria y el nivel de aprendizaje definido, 
tratando de mantener una coherencia entre los diferentes niveles y competencias. Esta iniciativa es destacable debido a que se trata de una aplicación realizada con el mundo productivo, consensuada y aplicada. Se deberá ir realizando un seguimiento para analizar los impactos y la efectividad en su utilización, pues uno de los sesgos que genera el levantamiento de marcos sectoriales (esto es por sectores productivos), sin un marco nacional de referencia, es que se tiende a relevar más el enfoque de gestión de recursos humanos al interior de las empresas y el sector específico, que las cualificaciones que permitirían la movilidad y el desarrollo de trayectoria laboral, ya sea horizontal o verticalmente.

\section{La visión de los actores relevantes en el medio nacional}

Otro elemento de base para el diseño de un marco de cualificaciones que incluya a los sistemas de capacitación laboral y de certificación de competencias lo constituye la visión de los actores considerados relevantes en el mundo del trabajo. Para ello, en el marco de este trabajo se elaboró una serie de entrevistas semiestructuradas a informantes clave, entre las que se incluyó a representantes de gremios empresariales, sindicatos, organismos de formación y profesionales especialistas. La síntesis de la observación realizada a partir de estas entrevistas se presenta a continuación.

Respecto de la utilidad o sentido que las personas entrevistadas le dan a la existencia de un marco, aparece la posibilidad de visualizar más claramente:

- las cualificaciones de las personas en el mundo del trabajo;

- el diseño y registro de las trayectorias laborales de los trabajadores;

- una focalización de los recursos destinados a inversión en capital humano por parte de las empresas, lo que podría significar mayor productividad;

- el ordenamiento de la oferta formativa, facilitando el diseño de los programas de estudios y permitiendo comparar planes y programas entre diferentes instituciones;

- la comparación de las cualificaciones de las personas 
independientemente de dónde hayan adquirido las competencias, tanto nacional como internacionalmente; $y$

- el diseño focalizado de programas que permitan acortar las brechas formativas, de experiencia laboral o de productividad.

En relación con los aspectos generales de la instalación de un marco de cualificaciones, en cualquier nivel, existió consenso respecto de los siguientes puntos:

a. Debiera tratarse como un tema independiente de los gobiernos de turno, debido a los plazos necesarios para su implementación.

b. Se requiere que algún ente institucional público lidere legítimamente, sin restar representación de otros actores.

c. Debe haber claridad respecto de la institucionalidad que rige el sistema y operar tripartitamente, haciendo sentido a los diferentes actores. Se debe estructurar sobre la base de instituciones y procesos que cuenten con la fe pública.

d. Debe existir una política pública que apoye el diseño y uso del marco de cualificaciones, lo que implica necesariamente abrir una discusión en torno a la calidad de la educación en los ámbitos que se relacionan con este marco.

e. Existen nudos legales e institucionales que deben removerse para generar los incentivos adecuados y funcionales a la instalación de un marco de cualificaciones que articule la formación laboral y la certificación de competencias.

f. Las actuales modalidades de financiamiento no proveen los recursos necesarios para fomentar la creación de un nuevo sistema regido por un marco de cualificaciones, el que necesariamente implica la formación por competencias, los sistemas de evaluación, la adecuación del perfil de los facilitadores y las adecuaciones tecnológicas para el registro de credenciales y visibilidad pública del sistema.

g. El diseño de un marco de cualificaciones parcial debe contemplar la posibilidad de articularse con un marco nacional de cualificaciones y con marcos de otros países, de manera de asegurar las posibilidades de homologación de cualificaciones.

h. Debe ser diseñado, en sus aspectos específicos, desde los distintos sectores productivos o económicos, con un compromiso decidido 
tanto de los gremios como de los sindicatos. Los sectores debieran estar representados por personas de alta influencia al interior de ellos, con participantes que tengan similar peso en las decisiones y fortaleciendo el rol de los trabajadores, ya que hoy es relativamente débil. La experiencia internacional muestra que todos los marcos de cualificaciones funcionan sobre la base de un modelo tripartito.

i. Debe irse adecuando a partir de una lógica acumulativa, es decir, preservar y valorar lo ya hecho, realizar el aprendizaje y construir sobre esa base.

Sobre la estructura del marco, los dos ámbitos relevantes son las dimensiones o descriptores que sirven para definir las características de una cualificación o nivel de aprendizaje y los niveles, que corresponden a las categorías o rangos de referencia que permiten agrupar las cualificaciones que se consideran equivalentes en función de determinados criterios, indicando cada uno la complejidad de los logros de aprendizaje que deben desarrollarse.

Respecto de las dimensiones que debiesen considerarse en un marco, pareciera existir consenso respecto de las dos primeras, que se refieren a conocimiento y habilidades. La tercera haría referencia a la relación de la persona con su entorno laboral y se asocia con descriptores como la autonomía relativa, la iniciativa, la responsabilidad en su trabajo y el de otros y la capacidad para resolver conflictos.

Acerca de los niveles, hay consenso en que se podría tener como referencia un marco nacional de ocho niveles, principalmente por ser esta la referencia del MEC (Marco Europeo de Cualificaciones) y que se ha comenzado a imponer como estándar. En ese contexto, un marco para la formación laboral podría llegar hasta el nivel cinco de un marco nacional. Esto considera un nivel 1 que responde al contexto específico del país y que incluye cualificaciones de ingreso al mundo laboral. El grueso de lo que hoy se entiende como formación laboral estaría localizado entre los niveles 2 y 4 , mientras los actuales perfiles laborales disponibles en ChileValora corresponderían a cualificaciones de nivel 2 o 3. 
Un punto de especial significación en la opinión de los actores relevantes para el funcionamiento de un marco lo constituyen las dificultades que observan para que efectivamente ello sea una realidad. $\mathrm{Al}$ respecto, mencionan que existe una baja capacidad de articulación entre los Ministerios de Educación, Trabajo y Economía, lo que sería un impedimento para trabajar en la construcción de un marco nacional.

En el mismo sentido operaría la normativa chilena, la que rigidiza las posibilidades de articulación. En la actualidad existen exigencias legales para poder cursar estudios superiores (necesidad de licencia media), lo que pone a un eventual diseño de marco en la disyuntiva de condicionar la estructura de niveles a dicha exigencia, o bien, buscar la flexibilización de la misma, lo que permitiría el reconocimiento (en credenciales) de cualificaciones que son reconocidas en el mundo del trabajo.

Un tercer elemento dice relación con una cultura formativa poco orientada al resultado del aprendizaje, lo que es consustancial a un sistema de reconocimiento sobre la base de las cualificaciones que se consiga acreditar. A ello se suma que no existe un sistema efectivo de supervisión y aseguramiento de la calidad de los procesos de trabajo.

\section{Principios y criterios para la generación de un marco de cualificaciones}

Tanto la experiencia revisada como la opinión de los actores relevantes permite formular a lo menos cuatro principios que debieran observarse al diseñar un marco de cualificaciones, particularmente a la hora de articular los sistemas de formación laboral y de certificación de competencias. Así, el marco de cualificaciones:

1. Debe ser reconocido por los actores del mercado como un instrumento que permite la implementación de itinerarios formativos y que orienta la movilidad de las personas en el mercado laboral.

2. Debe permitir la clasificación de las actividades profesionales (no reconocidas por un título en el sistema educativo) en un continuo de niveles que facilite los itinerarios formativos y las trayectorias laborales de las personas. 
3. Debe tener un diseño que permita posteriormente su integración en un marco de cualificaciones a nivel nacional.

4. Debe articularse con las experiencias nacionales desarrolladas a la fecha (en minería, SENCE e INACAP).

Concordantemente con estos principios que determinan el propósito del marco de cualificaciones se postulan criterios, los que son entendidos como las características que debe tener un nivel para constituirse en el «piso mínimo» de lo que se espera de la o las cualificaciones que contenga, buscando que cuenten con credibilidad y que hagan sentido tanto en el ámbito del trabajo como en el formativo.

Recogiendo las experiencias revisadas, tanto nacionales como internacionales, se definieron los siguientes criterios:

- Aplicabilidad: se deben considerar los aspectos legales y normativos que rigen en el contexto de la formación técnica, tanto formal como no formal.

- Amplitud: la matriz debe poder integrar la oferta de capacitación generada desde SENCE, los perfiles ocupacionales utilizados para la certificación de competencias (ChileValora) y, en un futuro, la oferta educacional. Asimismo, tanto la cantidad de niveles como los resultados de aprendizaje que los describan, deben considerar la cultura de la educación y capacitación propias del país.

- Pertinencia: los descriptores que se elijan y los resultados de aprendizaje que se definan para cada nivel deben tener en cuenta las competencias demandadas en el mundo laboral; tanto las de carácter técnico profesionales (conocimientos y habilidades), como las transversales (autonomía, responsabilidad, resolución de problemas, entre otras).

- Flexibilidad: los niveles y su descripción deben permitir que toda persona pueda avanzar y desarrollar nuevas cualificaciones, en una perspectiva de aprendizaje a lo largo de la vida, con lo que se posibilita la progresión horizontal (dentro de un mismo nivel) como la vertical (hacia niveles superiores).

- Portabilidad: las cualificaciones que integren la matriz y los resultados de aprendizaje que se describan para cada nivel 
deben considerar las características de la oferta educativa técnico profesional en sus distintos niveles y las credenciales que se pueden obtener en esta oferta, de manera que sean de utilidad a los usuarios y puedan ser reconocidas en diversos contextos.

Estos criterios pueden ser tomados como orientadores al momento de definir los descriptores o dimensiones que permitan diferenciar los niveles y describir los resultados de aprendizaje que se esperan para cada uno de ellos, teniendo como foco que los marcos «deben asegurar que las cualificaciones logren alcanzar las necesidades económicas y sociales del país, y que sean de calidad» (Tuck, 2007:13).

\section{Proyecciones}

Tanto la revisión de la experiencia internacional de orientación y propósito de los marcos de cualificaciones como la opinión de actores relevantes en el mundo del trabajo muestran que la utilización de este tipo de instrumento contribuiría a la articulación y ordenamiento de los sistemas de formación laboral y de certificación de competencias laborales financiados con recursos públicos en Chile.

Adicionalmente, los actores consideran que estarían dadas las condiciones para que se diseñe un marco de cualificaciones que apunte al objetivo señalado, aun cuando no exista un marco regulador a nivel nacional, el que requiere de otras articulaciones y diseños institucionales. No obstante, la existencia de este «submarco» podría operar como efecto demostrativo y dinamizador del proceso de construcción de una directriz nacional.

Para lograr la implementación del submarco descrito, se requiere cumplir con algunas condiciones relacionadas con los sistemas de capacitación laboral y certificación de competencias, entre las que se pueden mencionar el mejoramiento del catálogo de competencias, la consolidación de los procesos de evaluación y certificación de las competencias laborales, el alineamiento de los perfiles de competencias contenidos en el catálogo actual con la oferta formativa no formal, el desarrollo de estrategias de articulación con 
el sistema formativo formal y ampliar la información y difusión del sistema a los distintos sectores productivos (Eurosocial, 2012).

Un punto importante por lograr es conciliar los aspectos políticos y técnicos que están detrás del diseño y puesta en funcionamiento de un marco. Se trata de entender que un marco es un proceso dinámico. En efecto, si bien los marcos de cualificaciones tienen un componente técnico importante, cuentan también con un aspecto de carácter social y político tanto o más importante que radica en su usabilidad. Esta es la razón por la que se debe incorporar a estos procesos en los distintos grupos de interés (Lemaitre, 2010).

Junto con los actores relevantes (empresas, trabajadores y Estado), también se requiere de la voluntad política para generar la norma que permita la aplicación del marco de cualificaciones y genere incentivos para su uso. Como ejemplo, y en lo que se refiere a la articulación de la experiencia y formación adquirida en el mundo del trabajo con la formación técnico profesional, existen varias normas del ámbito de la educación que dificultan el reconocimiento de los aprendizajes previos o la articulación entre instituciones formativas, lo que no posibilita la homologación de credenciales y conformación de rutas formativas flexibles para las personas.

Finalmente y considerando el carácter pionero del diseño e instalación de este marco de cualificaciones para la formación laboral y la certificación de competencias, se hace fundamental convocar a distintos actores más allá del ámbito de las instituciones del trabajo, de tal manera que el proceso pueda ser seguido de cerca desde la institucionalidad de la educación y el fomento productivo, todos ellos actores públicos esenciales para la construcción futura de un marco nacional de cualificaciones.

\section{Referencias bibliográficas}

Alfonso, M.C. y F. Ferreira, (2007) «Vocational Education and Training in Portugal: Short Description», en CEDEFOP panorama series 144. [En línea]. Luxembourg, Office for Official Publications of the European Communities, disponible en: http://www.cedefop.europa.eu/EN/ Files/5177_en.pdf [Recuperado el 18 de noviembre de 2013]. 
Caramujo, E., (2009) «Quadro Nacional de Qualificações e a sua articulação com o Quadro Europeu de Qualificações», en Agência Nacional para a Qualificação [En línea]. Portugal, disponible en: http://www.crcvirtual. org/upload/imgs/Microsoft \%20PowerPoint\%20-\%20Cenfic\%20 13\%20Novembro\%2009.pdf [Recuperado el 18 de noviembre de 2013].

Centro Europeo para el Desarrollo de la Formación Técnica, CEDEFOP, (2010) «The Development of National Qualifications Frameworks in Europe», en CEDEFOP Working paper $N^{\circ} 8$ [En línea]. Luxembourg, Office for Official Publications of the European Communities, disponible en: http://www.cedefop.europa.eu/EN/Files/6108_en.pdf [Recuperado el 18 de noviembre de 2013].

Centro Europeo para el Desarrollo de la Formación Técnica, CEDEFOP, (2011) «The Development of National Qualifications Frameworks in Europe», en CEDEFOP Working paper $N^{\circ} 12$ [En línea]. Luxembourg, Office for Official Publications of the European Communities, disponible en: http://www.cedefop.europa.eu/EN/Files/6112_en.pdf [Recuperado el 18 de noviembre de 2013].

Centro Europeo para el Desarrollo de la Formación Técnica, CEDEFOP, (2012) «Analysis and Overview of NQF Developments in European Countries», en CEDEFOP Annual report 2012 Working paper $N^{\circ} 17$ [En línea]. Luxembourg, Office for Official Publications of the European Communities, disponible en: http://www.cedefop.europa.eu/EN/ Files/6117_en.pdf [Recuperado el 18 de noviembre de 2013]

Centro Microdatos, (2013) «Segundo estudio de competencias básicas de la población adulta 2013 y comparación Chile 1998-2013», en Corporación de Capacitación de la Construcción y Centro Microdatos. [En línea]. Santiago, disponible en: http://www.microdatos.cl/doctos_ noticias/Estudio\%20Competencias\%20Final_diseno.pdf [Recuperado el 18 de noviembre de 2013].

Consejo de Competencias Minero y Fundación Chile, (2013) «Marco de cualificaciones para la minería», en Consejo Minero [En línea]. Santiago, Consejo de Competencias Mineras, disponible en: http:// www.consejominero.cl/wp-content/uploads/2013/04/Marco-deCualificaciones-para-la-Mineria.pdf [Recuperado el 18 de noviembre de 2013].

Duarte, T., (2011) «EQF: The Key Lessons Learnt - the Portuguese Experience», en Agência Nacional para a Qualificação [En línea]. Portugal, disponible en: http://www.firstebtn.eu/files/final/EQF_\%20The\%20key\%20 lessons\%20learnt_\%20the\%20Portuguese $\% 20$ experience_Teresa $\% 20$ Duarte.pdf [Recuperado el 18 de noviembre de 2013]. 
Eurosocial, (2012) «Informe final. Análisis comparado de la situación actual del SNCP en los ocho países latinoamericanos: Brasil, Chile, Colombia, Ecuador, El Salvador, Paraguay, Perú y Uruguay», en Programa Eurosocial [En línea]. Disponible en: http://media.wix.com/ugd/a99 e24_76e7031a7de62447796bc0a51b16a249.pdf [Recuperado el 18 de noviembre de 2013]

Kis, V. y Field, S. (2009) «Learning for Jobs. OECD Reviews of Vocational Education and Training. Chile: A first report». OECD 2009.

Lemaitre, M. J., (2010) «Desarrollo de un Marco Nacional de Cualificaciones para el Sistema Educacional de Chile. Lecciones de la Experiencia. Informe Final Proyecto MECESUP UCN 0701: Bases para el diseño de un Marco de Cualificaciones, Títulos y Grados para el Sistema de Educación Superior Chileno». Documento sin publicar. MECESUP UCN, 2010

Organization for Economic Co-operation and Development, OECD, (2013) OECD Economic Surveys: Chile 2013. OECD Publishing. doi: 10.1787/eco_surveys-chl-2013-en

PNUD, (2011). «Informe final, comisión revisora del sistema de capacitación e intermediación laboral», en Ministerio del Trabajo de Chile [En línea]. Santiago. Disponible en: http://www.mintrab.gob.cl/?p=5387 [Recuperado el 18 de noviembre de 2013]

Programa de Mejoramiento de la Calidad y Equidad de la Educación, MECESUP, Bicentenario. (2009) «Diseño de un marco de cualificaciones para el sistema de educación superior chileno», en Consejo de Rectores [En línea]. Santiago, disponible en: http://www.consejoderectores.cl/ innovacion_curricular/marco_cualificaciones/documentos/Revista_ Mc.pdf [Recuperado el 18 de noviembre de 2013]

Programa Chilecalifica - Universidad Federico Santa María, (2010) «Marco Nacional de Cualificaciones aplicado al Sector Minería. Informe final». Chile. Documento sin publicar. Programa Chile Califica, 2010.

Organization for Economic Co-operation and Development, OECD, (2013) OECD Economic Surveys: Chile 2013. OECD Publishing. doi: 10.1787/eco_surveys-chl-2013-en

Somavía, J., (1999) «Trabajo decente: memoria del director general», en Organización Internacional del Trabajo [En línea]. Ginebra, disponible en: http://www.ilo.org/public/spanish/standards/relm/ilc/ilc87/rep-i. htm [Recuperado el 18 de noviembre de 2013]

Strathdee, R., (2010) «The Implementation and Impact of the New Zealand», en National Qualifications Framework, ILO. [En línea]. Nueva Zelanda, disponible en: http://www.ilo.org/wcmsp5/groups/public/---ed_ 
emp/---ifp_skills/documents/genericdocument/wcms_145930.pdf [Recuperado el 18 de noviembre de 2013]

Tuck, R., (2007) «An Introductory Guide to National Qualifications Frameworks. Conceptual and Practical Issues for Policy Makers», en International Labour Organization [En línea]. Suiza, disponible en: http://www.ilo.org/wcmsp5/groups/public/---ed_emp/---ifp_skills/ documents/instructionalmaterial/wcms_103623.pdf [Recuperado el 18 de noviembre de 2013]

Vargas, F. y N. Billorou, (2010) Herramientas básicas para el diseño e implementación de marcos de cualificaciones. Guía de trabajo. Uruguay, CINTERFOR-OIT.

Recibido: 25/11/2013

Aceptado: 29/11/2013 